Journal of Engineering Science and Technology Review 5 (3) (2012) 10-19

Special Issue on Simulation of Manufacturing Technologies

Research Article

\title{
Numerical Simulation of Friction Welding Processes Based on ABAQUS Environment
}

\author{
Wenya Li*, Shanxiang Shi, Feifan Wang, Zhihan Zhang, Tiejun Ma and Jinglong Li \\ State Key Laboratory of Solidification Processing, Shaanxi Key Laboratory of Friction Welding Technologies, \\ Northwestern Polytechnical University, Xi'an 710072, Shaanxi, PR China
}

Received 15 June 2012; Accepted 8 November 2012

\begin{abstract}
Friction welding $(\mathrm{FW})$ is a collection of a series of friction-based solid state joining processes which can produce high quality welds of different components with either similar or dissimilar materials and has been attracting increasing attention. Due to the extreme condition encountered during FW and the highly thermomechanical coupled nature of FW, finite element methods have been widely developed to study the FW process. In the light of reasonable simplification, we developed several effective methods based on the ABAQUS environment. Initially 2D models were developed to investigate the complete FW process, where an implicit method with remeshing and map solution techniques and an explicit method with the Arbitrary Lagrangian Eulerian (ALE) adaptive mesh controls were proposed to effectively overcome the excessive element distortion by using the ABAQUS Standard and Explicit packages, respectively. In addition, a 3D model was also developed to obtain better simulation results with the help of the ALE adaptive mesh controls in the ABAQUS/Explicit package and the map solution technique in the ABAQUS/Standard package combined with the HYPERWORKS software. The experiments validate the feasibility and accuracy of the developed models.
\end{abstract}

Keywords: Friction welding, Rotary friction welding, Linear friction welding, Friction stir welding, Numerical simulation

\section{Introduction}

Friction welding $(\mathrm{FW})$ is a collection of a series of friction-based solid state joining processes, in which the frictional heat generated via relative movement between two components under a force is used to soften the interface material, and at some time when the material has softened sufficiently the forging force is applied to form the joint. These techniques show considerable advantages when compared to traditional fusion welding techniques, such as the absence of solidification defects, ability to weld dissimilar materials, lack of consumables, ease of automation, limited energy requirements and high ecocompatibility. Therefore, FW has been attracting increasing attention in many applications, such as aerospace, automobiles, railway and nuclear industry.

Among those FW techniques, the more interesting processes are:

(1) Rotary friction welding (RFW) [1], in which cylindrical parts, typically rods or tubes, are placed in rotation with each other and subjected to an axial compression load, as illustrated in Fig. 1. The continuous-drive friction welding (CDFW) and inertia friction welding (IFW) are the representative RFW processes.

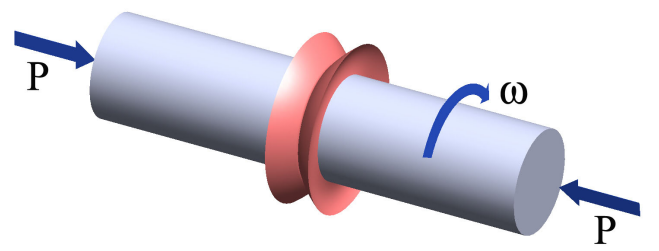

Fig. 1. Rotary friction welding.

(2) Linear friction welding (LFW) [2], in which reciprocal linear movement of one component relative to the other is applied to produce heat, as illustrated in Fig. 2. LFW represents the evolution of RFW for non-axisymmetric parts and has been successfully used in manufacturing aeroengine blisks.

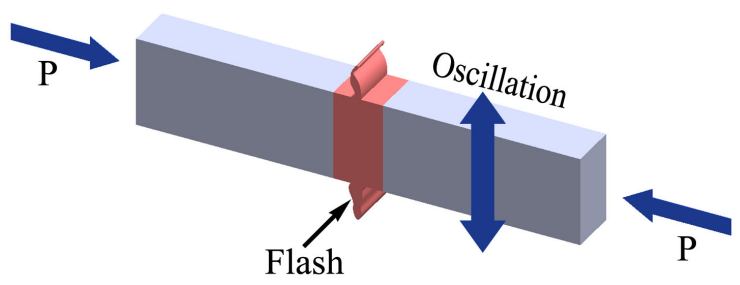

Fig. 2. Linear friction welding.

(3) Friction stir welding (FSW) $[3,4]$, a technique that 
involves using an appropriate non-consumable stir tool for the heating and mixing of the contact surfaces of metal sheets to be welded, as illustrated in Fig. 3.

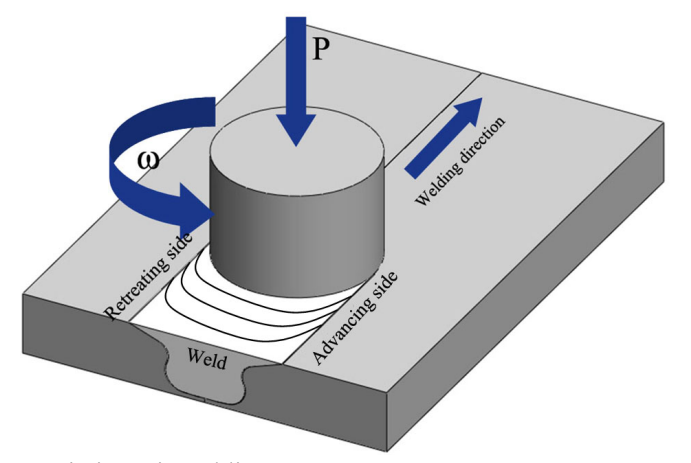

Fig. 3. Friction stir welding.

Since their emergence, numerous experiments have been done to develop their industrial applications by optimizing processing parameters and joint properties. However, the understanding of their nature is to a large extent hindered due to the extreme conditions encountered in FW and the highly thermomechanical coupled process of FW. Therefore, the finite element method has also been increasingly employed to study the FW process and particularly the joint appearance, as well as temperature history, strain and stress fields, and for even microstructure and property predictions.

The CDFW process was modeled at the beginning by investigators because it is the oldest FW process. Healy et al. [5] and Bendzsak et al. [6] analyzed the steady stage of CDFW by the numerical method. The analytical results were to some extent in agreement with experiments $[5,6]$. Midling and Grong [7] investigated the temperature evolution and plastic flow of joints during the overall CDFW stages by numerical simulations and experiments. They categorized different regions of joints as: the contact zone at the joint interface; the fully plasticized region where the deformation depends on a combination of the rotational velocity gradient and the rates of axial and radial upsetting; the partly deformed region; and the undeformed base material [7]. Sluzalec [8] developed a finite element method code computing strain and stress fields in the welded components.

With the help of numerical process modelling, the effects of welding parameters during IFW were explored. A few IFW models have been described in literature, but most of them do not include the rotational motion of the process. Davé et al. [9] built the only analytical thermal model for IFW found in literature. They predicted the transient thermal profiles of IFW tubes but did not consider material deformation and flow during the forging stage of IFW. A number of assumptions were made, such as the average temperature across the wall thickness and material properties were averaged for the entire temperature range. Even though this model presented a very simplified approach, reasonable agreement was found between thermocouple measurements and temperature predictions. Soucail and Bienvenu [10] made one of the first attempts to fully predict the IFW process using a $2 \mathrm{D}$ coupled thermomechanical finite element (FE) model. As with many models, it does not consider rotational motion of the IFW process. Validation of the temperature predictions was performed using a pyrometer and thermocouple measurements, as well as metallographic techniques. Good agreement in the thermal profiles was found close to the weldline. D'Alvise et al. [11] produced a similar 2D coupled thermomechanical FE model. The noticeable aspect of this model was that it underestimated the welding time and upset, but showed good correlation with thermocouple data for the first 4 seconds of the welding process. Zhang et al. [12] created a 2D coupled thermomechanical model using for the first time an elastoplastic formulation. Reasonable correlation was found with thermocouple data, however no comparison was made to the burn-off rate, which makes it difficult to judge how accurately their model predicted material flow. Bennett et al. [13] produced a model using the commercial code Deform $2 \mathrm{D}$ attempting to fully simulate IFW by initially training the heating model using data from previously performed welds. This model considers the rotational motion by using a 2.5 dimensional element to model plane strain. Results of the upset prediction were only shown for the training phase of the model with an error of about $10 \%$. Thermal profiles at 1 $\mathrm{mm}$ away from the final weldline were within $10 \%$ of the actual values, however further away from the weldline the difference increased. In contrast, Wang et al. [14] produced a coupled thermomechanical model within Deform 2D using weld trial data (such as torque, angular rotation speed and upset) to determine the rate of energy input and forging rate as imposed boundary conditions. The temperature predictions of the model were validated by comparing microstructural changes within the heat-affected zone of the weld with thermodynamical prediction of the microstructure for certain peak temperatures and microstructural observations in rapidly heated samples. Generally good agreement was found between experimental and predicted data, although the model underestimated the temperature gradient in the axial direction. But they further modified their model to predict residual stresses and acceptable results were obtained [15]. It should be emphasized that their modified model did not predict welding parameters, but rather used data recorded during welding trials to obtain a better insight into the thermomechanical history the material undergoes during IFW [15].

As for LFW, because of the limited applications (e.g. blisks) and high machine cost, few studies have been presented, and especially numerical simulation work. Vairis and Frost [16] established a numerical model and analyzed the thermo-mechanically coupled relationship to obtain temperature history in the initial stage of LFW. SorinaMüller et al. [17] developed a 3D thermo-mechanical model for LFW of Ti-6Al-2Sn-4Cr-6Mo using ANSYS to explore temperature distribution in the rubbing interface. Turner et al. [18] formulated a 2D finite element model for LFW of Ti$6 \mathrm{Al}-4 \mathrm{~V}$ based on FORGE. They found that the simulated temperature corresponds to the measured one and that the highest temperature reached at the interface was about $1100^{\circ} \mathrm{C}$. Tao et al. [19] studied a thermo-mechanically coupled model using DEFORM for LFW of Ti-6Al-4V. They found that the highest temperature reached was about $1340^{\circ} \mathrm{C}$ and appeared at the centre of the specimen, which corresponds to experiments. Li et al. [20] produced a 2D thermo-mechanically coupled model for LFW of Ti-6Al-4V using ABAQUS. The effects of processing parameters were examined and temperature contours in the specimen at different friction times were estimated.

FSW being the latest innovative but most complex process has been widely applied to weld lightweight alloys, such as aluminum and magnesium alloys, and more recently, titanium alloys, copper alloys, steels and superalloys $[3,4]$. Sound welds are achieved only for specific combinations of parameters that have almost exclusively been obtained through extensive experiments, and the particular 
phenomena that are responsible for joining are not fully understood yet. Numerical simulations are helpful to better understand and observe the influence of input parameters on the resulting phenomena, to visualize and analyze the temperature field and material flow. The first models reported in the literature are analytical and address temperature analysis. Initially based on Rosenthal's equations, they describe the quasi-steady temperature field of a semi-infinite body on which a surface heat source moving with a constant velocity is applied. Gould and Feng [21] incorporated in their equations the term of frictional heat, assuming a constant uniform pressure between the tool and the workpieces. The traverse speed of the tool, the rotational velocity and the loading force were the three parameters of the model. Schmidt et al. [22] proposed an analytical expression for estimating heat generation for more complex tool shapes. Thereafter, the convective heat transfer due to material flow in the shear layer was taken into account by prescribing a velocity boundary condition for the convective term in the energy equation. Khandkar et al. [23] introduced a torque-based heat input model in which the local heat flux is linearly related to the distance to the tool axis. All these models constitute the first approaches to approximate the temperature map or the heat source in FSW. However, they are based on strong assumptions concerning contact, by assuming pure sliding or sticking conditions, and always need the adjustment of experimental coefficients to be accurate.

When pure Lagrangian formulations are used, the high distortions of the mesh are the main difficulty that FE models have to deal with. They occur under the tool shoulder, where high strains are logically observed during FSW. To overcome this the FSW tool is usually substituted by an analytical heat source. The simulation of material flow during FSW has been modeled using computational fluid dynamics (CFD) formulations, the material being regarded as a viscous fluid flowing across an Eulerian mesh and interacting with a rotating tool. In the model developed by Ulysse [24], the contact was assumed to be sticking. Consequently, the heat source is only due to the viscous dissipation of the laminar flow. Seidel and Reynolds [25] approximated the viscosity by a function of the material yield stress throughout the Zener-Hollomon parameter. Their model was implemented with the 3D code FLUENT. Nandan et al. [26] also produced the 3D CFD model of FSW of mild steel using spatially variable thermophysical properties and a methodology adapted from well-established previous work in fusion welding. Non-Newtonian viscosity or the metal flow was calculated considering temperature and strain rate dependent flow stress. The predicted temperature against time plots agreed well with experimental results. Colegrove et al. [27] also used a CFD code to develop a global thermal model in which the heat flow, which is applied at the tool/workpiece interface, includes terms due to material shearing and friction. They also developed a local model to visualize the influence of the screw on the material flow around the probe. In the approach of Cho et al. [28], the evolution equation for strain hardening of the material was handled and integrated along the streamlines of the flow field. The major weakness of these CFD like models is the approximations which result from the material rheology, which generally does not allow for residual stresses prediction, and from the friction contact surface. In addition, the simulations of transient phases and defects of the process are very difficult. On the other hand, the arbitrary Lagrangian-Eulerian (ALE) description makes it possible to take into account movements of free surfaces while reducing mesh distortions. This formulation procures the best compromise between Lagrangian and Eulerian formulations. Therefore, a 3D numerical tool enabling the thermo-mechanical simulation of the whole FSW process (plunging phase, dwelling phase and welding phase) has been developed [29]. But the ALE method has still the limitation in coping with the real stirring process of FSW.

\section{Methods}

In recent years, we employed a commercial FE code, the ABAQUS software [30], to develop the simulation models to analyze the FW process based on its robust capabilities to solve transient thermal or thermomechanical problems. Some special computational techniques were used in these models to deal with the peculiarities of FW as described in the following sections.

\subsection{Contact model, heat generation and heat input}

Usually, contact in FW is considered to follow the conventional Coulomb friction law, i.e., the shear stress of the contacting interface is expressed as follows:

$\tau_{\text {fric }}=\mu P$

where $\tau_{\text {fric }}$ is the friction shear stress, $\mu$ the friction coefficient and $\mathrm{P}$ the normal contact pressure. Hence, the heat generation from the friction is described as follows:

$$
q_{\text {fric }}=\eta \dot{\gamma} \tau_{\text {fric }}=\eta \mu P \dot{\gamma}
$$

where $\dot{\gamma}$ is the slipping rate, which may be dependent on the relative position in different $\mathrm{FW}$ processes having various movement forms, $\eta$ the heat conversion efficiency. The friction coefficient could be a variable dependent on the interface temperature, relative slipping rate between the two faying surfaces, and normal pressure. However for FW the conventional Coulomb friction law will be only applied at the very beginning of welding when interface temperature is relatively low. As the interface plasticized material is formed in larger volumes at elevated temperatures, the friction behavior will be dominated by viscoplastic friction. Therefore, heat generation is dependent on intense plastic deformation of the thin shear layer at the interface. A modified Coulomb friction law is then applied [31], where the equivalent flow stress of the material is used as follows:

$$
\tau_{\text {fric }}=\tau_{\text {shear }}=\sigma_{s} / \sqrt{3}
$$

where $\tau_{\text {shear }}$ is the flow shear stress calculated from the equivalent flow stress $\sigma_{\mathrm{s}}$. Hence the heat generation (q) during FW could be expressed as follows:

$$
q=\eta \dot{\gamma} \min \left(\mu P, \sigma_{s} / \sqrt{3}\right)
$$

In most circumstances, frictional heat at the very beginning is used to create the initial temperature field and is followed by heat generation from plastic deformation. In other words, according to friction theory the conventional friction behavior could be taken as the local plastic deformation of asperities of the contacting surfaces, then heat generation during FW is defined as: 


$$
q=\eta \dot{\gamma} \sigma_{s} / \sqrt{3}
$$

This assumption has been proved to be effective [32].

Some researchers have also considered parts of heat generation coming from sliding friction and sticking shear during FSW as follows:

$$
q=(1-\delta) \eta \dot{\gamma} \sigma_{s} / \sqrt{3}+\delta \eta \dot{\gamma} \mu P
$$

where $\delta$ is the fraction of heat generated from sliding friction.

\subsection{Boundary conditions}

Normally, heat flux boundary conditions could be determined from the above mentioned heat generation models to assume as input the surface heat source from the interface or the body heat source from the interfacial zone (shear layer).

As for convection boundary conditions, these are heat losses from the workpiece surfaces exposed to air, from radiation, and from the fixture or the tool (FSW) via thermal contact. The first two convection losses are common and can be found in literature. Concerning the heat loss from the fixture, e.g. clamps used in RFW or LFW, and backplates used in FSW, an equivalent convective coefficient was assumed for heat loss, which could be a function of temperature and position. While for heat loss from the tool (FSW), the total heat generated at the tool-workpiece interface has been split by a ratio (f) to the workpiece according to their thermal properties:

$$
f=\frac{\sqrt{\left(k \rho C_{p}\right)_{\text {workpiece }}}}{\sqrt{\left(k \rho C_{p}\right)_{\text {workpiece }}}+\sqrt{\left(k \rho C_{p}\right)_{\text {tool }}}}
$$

where $\mathrm{k}, \rho$ and $\mathrm{C}_{\mathrm{p}}$ are material thermal conductivity, density and heat capacity, respectively. The subscripts "workpiece" and "tool" stand for the properties of workpiece and tool, respectively.

\subsection{ALE adaptive meshing and mesh-to-mesh solution mapping}

Three types of adaptivity techniques are available in ABAQUS that can optimize a mesh and maintain a highquality mesh throughout an analysis : the ALE adaptive meshing, the adaptive remeshing varying topology, and the mesh-to-mesh solution mapping to enable rezoning analysis. In previous studies on FW, ALE adaptive meshing and mesh-to-mesh solution mapping were used.

The ALE adaptive meshing used in ABAQUS/Explicit, provides control of mesh distortion in cases where large deformation occurs. ALE adaptive meshing uses a single mesh definition that is gradually smoothed within analysis steps. The term ALE implies a broad range of analysis approaches, from purely Lagrangian analysis, in which the node motion corresponds to material motion, to purely Eulerian analysis, in which the nodes remain fixed in space and material "flows" through the elements. Typically ALE analyses use an approach between these two cases.

The mesh-to-mesh solution mapping is available only in ABAQUS/Standard. One can use this technique to control element distortion by replacing the mesh and continuing the analysis after solution mapping. Mesh replacement, or rezoning, involves the creation of multiple ABAQUS jobs, each of which represents the configuration of the model in distinct, sequential periods of the simulation history. One uses mesh replacement when a single mesh cannot be effective for the duration of a simulation. Each mesh change after the initial configuration reflects a solution-dependent deformed configuration of the model. Therefore, analyses that use mesh replacement are sequentially dependent, and ABAQUS uses mesh-to-mesh solution mapping to propagate solution variables from one analysis to the next. In contrast to adaptive remeshing, each mesh replacement job represents a component of the overall analysis history. The first requirement for remeshing is some indication that the mesh is becoming distorted in regions where this distortion could cause the solution to be inaccurate. One possible criterion for remeshing would be extreme element distortion in areas where high strain gradients need to be resolved accurately. Ultimately, however, the decision to remesh is a matter of judgment depending on specifics.

\subsection{Mass scaling}

Mass scaling is often used in Abaqus/Explicit for computational efficiency in quasi-static or dynamic analyses that contain a few very small elements that control the stable time increment. The explicit dynamics procedure is typically used to solve two classes of problems: transient dynamic response calculations and quasi-static simulations involving complex nonlinear effects. Because the explicit central difference method is used to integrate the equations in time, the discrete mass matrix used in the equilibrium equations plays a crucial role in both computational efficiency and accuracy for both classes of problems. When used appropriately, mass scaling can often improve the computational efficiency while retaining the necessary degree of accuracy required for a particular problem class. However, the mass scaling techniques most appropriate for quasi-static simulations should be carefully used for dynamic analyses because the natural time scale is generally not important for quasi-static simulations, while the natural time scale is always important in dynamic analysis, and an accurate representation of the physical mass and inertia in the model is required to capture the transient response. By scaling the masses of the small controlling elements at the beginning of the step, the stable time increment can be increased significantly, yet the effect on the overall dynamic behavior of the model may be negligible. We had made many trials on mass scaling adjustment and obtained the suitable values for FW.

\subsection{Programming and subroutines}

In the ABAQUS development environment, the ABAQUS scripting interface is an application programming interface (API) to the models and data used by ABAQUS, which is an extension of the Python object-oriented programming language, and can be used to create and modify the components of an ABAQUS model, such as parts, materials, loads, and steps; to create, modify, and submit ABAQUS analysis jobs; to read from and write to an ABAQUS output database; to view the results of an analysis. Therefore, this technique is very useful for automation of computation, post-processing and analysis of simulated results.

On the other hand, plenty of user subroutines and utility routines available in ABAQUS facilitate the treatment of the complex behaviour of the process. The user subroutines based on FORTRAN are used to define the specific phenomena which are not available in the standard ABAQUS procedures, such as user defined friction 
behaviors, user defined material constitutive models and user defined boundary conditions and loads. The utility routines can be used within user subroutines to perform a variety of common tasks, such as obtaining the solution information, printing the important messages and terminating an analysis.

\subsection{Material model}

The Johnson-Cook material model was used, describing the flow stress $\sigma$ as a function of strain hardening, strain rate hardening and temperature softening as below.

$$
\sigma=\left(A+B \varepsilon^{n}\right)\left[1+C \ln \left(1+\frac{\dot{\varepsilon}}{\dot{\varepsilon}_{0}}\right)\right]\left(1-\left[\frac{T-T_{\text {room }}}{T_{\text {melt }}-T_{\text {room }}}\right]^{m}\right)
$$

where $\mathcal{\varepsilon}$ and $\dot{\mathcal{E}}$ are strain and strain rate, respectively, $\dot{\mathcal{E}}_{0}$ the reference strain rate, $T_{\text {room }}$ the reference temperature, $T_{\text {melt }}$ the melting point. $A, B, n, C, m$ are constants dependent on the material.

To cope with the abnormal element distortion, the Johnson-Cook damage model was tried in the simulation. Fracture in this model is derived from the cumulative damage law as follows [30]:

$$
D=\Sigma\left(\Delta \varepsilon / \varepsilon_{\text {failure }}\right)
$$

here, $D$ is the failure parameter, and failure is assumed to occur when $D$ equals to 1.0 . The current failure strain is defined as:

$$
\varepsilon_{\text {failure }}=\left\lfloor D_{1}+D_{2} \exp \left(D_{3} \sigma^{*}\right)\right] \cdot\left[1+D_{4} \ln \left(\dot{\varepsilon}^{*}\right)\right]\left(1+D_{5} T^{*}\right)
$$

where, $\Delta \varepsilon$ is the increment of effective plastic strain during an increment in loading and $\sigma^{*}$ the mean stress normalized by the effective stress. The parameters, $D_{1}, D_{2}, D_{3}, D_{4}$ and $D_{5}$ are material constants.

\section{Case studies}

\subsection{Case 1: CDFW [32]}

\section{Case description:}

The commonly used 20\# low carbon steel, with a composition of $0.17-0.24 \mathrm{wt} \% \mathrm{C}, 0.17-0.37 \mathrm{wt} \% \mathrm{Si}$ and 0.35 $0.65 \mathrm{wt} \% \mathrm{Mn}$, was CDFWed in the experiment. The mild steel rods have a length of $150 \mathrm{~mm}$ and diameter of $20 \mathrm{~mm}$. The welding parameters are: the rotating speed, axial pressure, forging pressure, friction time and forging time of $1200 \mathrm{rpm}, 200 \mathrm{MPa}, 400 \mathrm{MPa}, 3.5 \mathrm{~s}$ and $0.1 \mathrm{~s}$, respectively.

\section{Geometry and model:}

According to heat transfer theory, when a semi-infinite specimen has unsteady heat conduction the affected depth $x$ is estimated by:

$$
x=4 \sqrt{a t}
$$

where, $a$ is the heat transfer coefficient and $t$ the time. Hence, $40 \mathrm{~mm}$ length is adequate for this simulation case to reduce the amount of elements and computation time. The 2D axisymmetric model was established in ABAQUS/Standard as shown in Fig. 4. As for meshing, the specimen was partitioned into two regions, where the upper $34 \mathrm{~mm}$ has a meshing size of $2 \mathrm{~mm}$ and the lower $6 \mathrm{~mm}$ has a meshing size of $0.8 \mathrm{~mm}$ (Fig .1). Due to the extensive interface deformation in the CDFW process, the mesh-to-mesh solution mapping technique was engaged to overcome the abnormal element distortion. The computation was monitored by a Python code to automatically remesh and apply map solution.

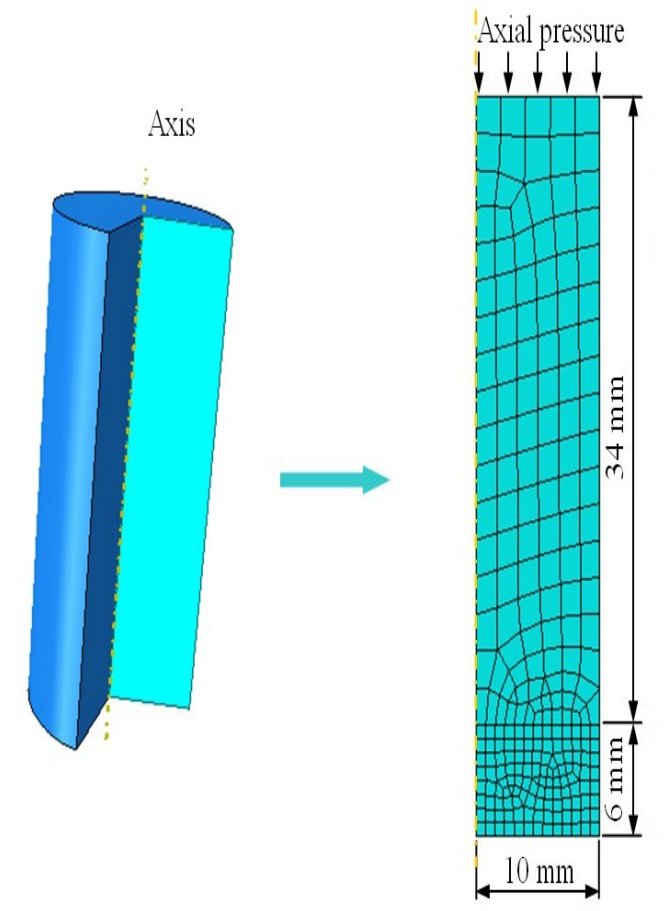

Fig. 4. 2D axisymmetric model and mesh for CDFW of mild steel rods.

Heat generation at the interface was defined as a heat flux through the subroutine DFLUX available in ABAQUS, which is determined by rotating speed, axial pressure and/or shear flow stress. Here, both the effects of the nonuniform linear velocity along the radial direction of specimen and pressure distribution across the interface were taken into account.

\section{Results and discussion:}

The typical simulation result is obtained under the same welding parameters as in the experiment. The transient temperature fields at different welding times are shown in Fig. 5. It can be seen that the interface temperature rises sharply to about $1000^{\circ} \mathrm{C}$ within $0.1 \mathrm{~s}$. With increasing welding time, the high temperature zone widens from the weld interface due to the heat conduction within the specimen. Moreover, the maximum interface temperature presents a quick increase to a quasi-stable temperature phase with a slight fluctuation when the temperature becomes higher than about $1100^{\circ} \mathrm{C}$ (Fig. 6). It was also found that flash is not present at $1.5 \mathrm{~s}$, but the volume of flash begins to increase with welding time from $1.5 \mathrm{~s}$ to $3.5 \mathrm{~s}$ and the largest flash is obtained after the forging process. Finally, the largest axial shortening (about $7.7 \mathrm{~mm}$ ) is also obtained (Fig. 6). During the short forging period, the interface temperature sharply decreases with the extrusion of high temperature thermoplastic material. 


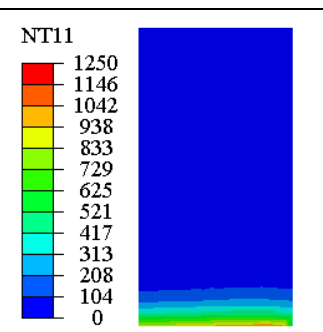

$\mathrm{t}=0.1 \mathrm{~s}$

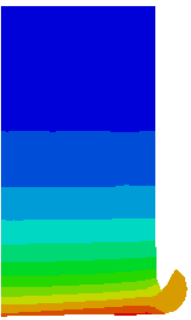

$\mathrm{t}=2.5 \mathrm{~s}$

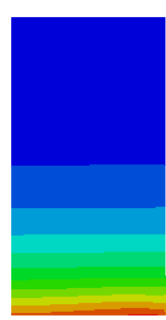

$\mathrm{t}=1.5 \mathrm{~s}$

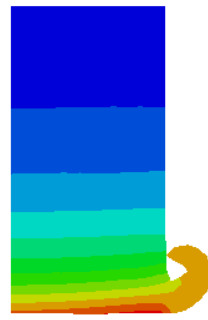

$\mathrm{t}=3.5 \mathrm{~s}$

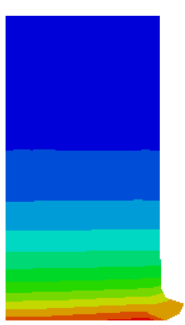

$\mathrm{t}=2.0 \mathrm{~s}$

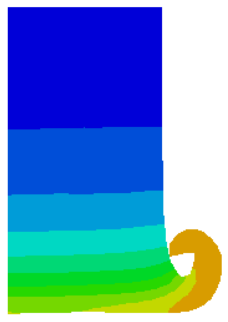

$\mathrm{t}=3.6 \mathrm{~s}$
Fig. 5. Temperature contours at different welding times.

Through measuring the dimensions of the specimens before and after experiment, the axial shortening was obtained as about $7.5 \mathrm{~mm}$, which means an error of $2.5 \%$ between experimental and computed axial shortening indicating a good agreement between experiment with simulation. Fig. 7 shows the comparison of the final crosssection between the calculated result (Fig. 7a) and experiments (Fig. 7b). It can be found that the simulated flash shape is similar to experiments. In addition, the width of the high temperature zone in the vicinity of the weldline is also comparable. The microstructure observed indicates that the simulated temperature distribution is comparable to the experiment [32]. Therefore, the developed model demonstrates the feasibility to simulate the CDFW process.

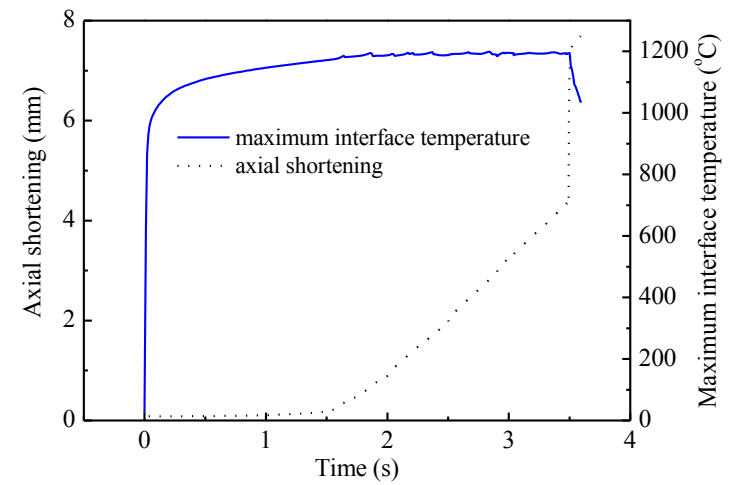

Fig. 6. Evolutions of the maximum interface temperature and axial shortening during CDFW of mild steel.

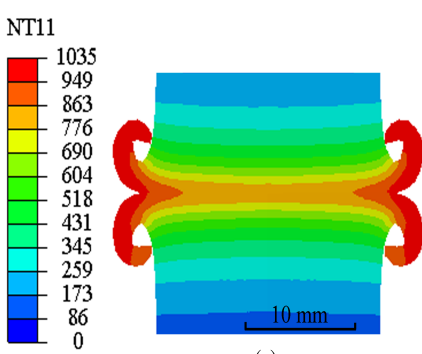

(a)

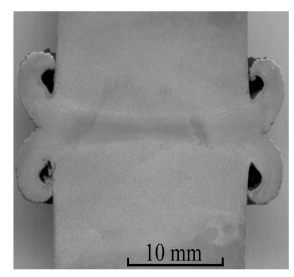

(b)
Fig. 7. Comparison of final cross-sections between simulation (a) and experiment (b).

\subsection{Case 2: IFW [33]}

The new generation high performance superalloy FGH96, which has a chemical composition of $2.49 \mathrm{wt} \% \mathrm{C}$, $16.37 \mathrm{wt} \% \mathrm{Cr}, \quad 13.0 \mathrm{wt} \% \mathrm{Co}, \quad 4.88 \mathrm{wt} \% \mathrm{~W}, \quad 4.83 \mathrm{wt} \% \mathrm{Mo}$, $1.93 \mathrm{wt} \% \mathrm{Al}, \quad 3.46 \mathrm{wt} \% \mathrm{Ti}, \quad 0.25 \mathrm{wt} \% \mathrm{Zr}, \quad 0.74 \mathrm{wt} \% \mathrm{Nb}$ and $52.06 \mathrm{wt} \% \mathrm{Ni}$, was IFWed in the experiment. The superalloy tubes had a length of $20 \mathrm{~mm}$, inner diameter of $40 \mathrm{~mm}$ and wall thickness of $10 \mathrm{~mm}$. The welding parameters are: the initial rotational speed, axial pressure and moment of flywheel of $750 \mathrm{rpm}, 390 \mathrm{MPa}$ and $35.9 \mathrm{~kg} \cdot \mathrm{m}^{2}$, respectively.

\section{Geometry and model:}

Similar to the model of CDFW, a 2D axisymmetric model was built for IFW, as shown in Fig. 8, based on the same specimen size as in the experiment. The mesh was created using the quad-dominated, coupled displacementtemperature elements with the twist degree of freedom. The mesh size was chosen to change over the length of the specimen, as shown in Fig. 8, to reduce computation time while maintaining accuracy of the results. Due to the extensive interfacial deformation in the IFW process, the mesh-to-mesh solution mapping technique was used to overcome excessive element distortion. The self-contact was also utilized to avoid early simulation abortion.

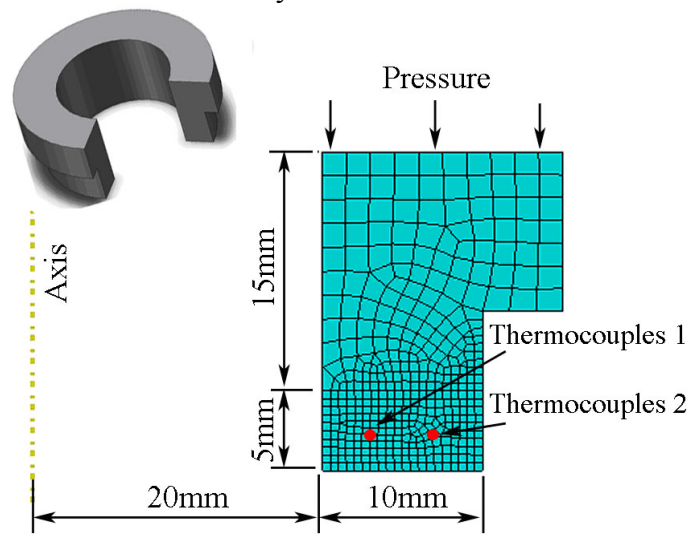

Fig. 8. Geometric model and mesh of $2 \mathrm{D}$ axisymmetric model.

A similar heat generation to CDFW at the interface was adopted as a heat flux through the subroutine DFLUX available in ABAQUS, which is determined by the transient flywheel rotational speed, axial pressure and/or shear flow stress. Here, both the effects of the non-uniform linear velocity along the radial direction of specimen and the pressure distribution across the interface were taken into account.

\section{Results and discussion:}

The comparison between experimental and simulated temperatures is shown in Fig. 9. Thermocouples were placed $2.5 \mathrm{~mm}$ away from the initial weld interface and $3 \mathrm{~mm}$ away from inner (point 1) and outer (point 2) walls as shown in Fig. 8. It can be clearly seen that the simulated temperatures are similar to experiments except for the damage of thermocouples at $4.3 \mathrm{~s}$. The temperature reached could be higher than $1000^{\circ} \mathrm{C}$ during IFW. The simulated widths of high temperature zones $(\mathrm{a}, \mathrm{b}$ and $\mathrm{c}$ are temperatures over $750^{\circ} \mathrm{C}, 1030^{\circ} \mathrm{C}$ and $1130^{\circ} \mathrm{C}$, respectively) during IFW are shown in Fig. 10. As the working temperature of FGH96 is about $750^{\circ} \mathrm{C}$, the region having a temperature above $750^{\circ} \mathrm{C}$ is defined as the heat-affected zone (HAZ). Moreover, increasing the working temperature the precipitation peak of $\gamma^{\prime}$ takes place at about $1030^{\circ} \mathrm{C}$, while $\gamma^{\prime}$ will completely 
dissolve at a temperature above $1130^{\circ} \mathrm{C}$. It can be found that the widths of $b$ and $c$ rise quickly to reach an equilibrium, while the size of the HAZ shows another pronounced increase right after the stop of the flywheel due to heat conduction from the high temperature interfacial zone. The maximum width of zone is about $8 \mathrm{~mm}$.

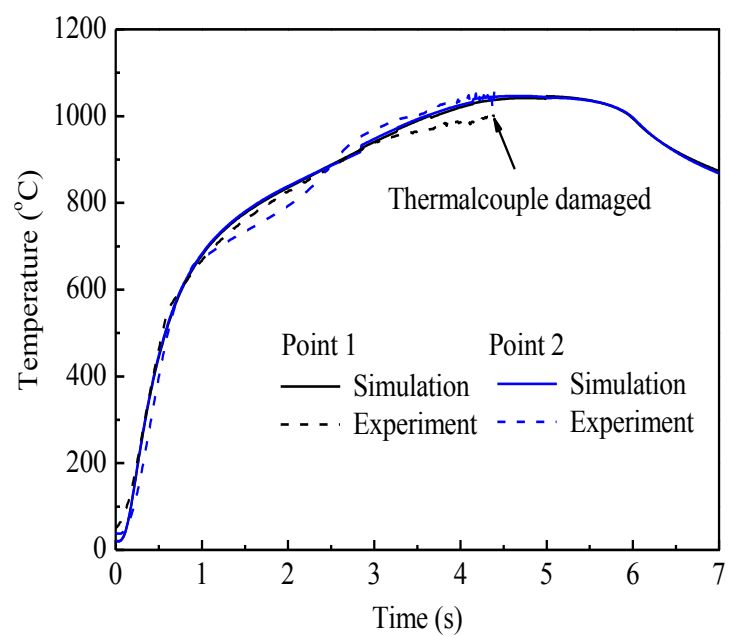

Fig. 9. Comparison of experimental and simulated temperatures.

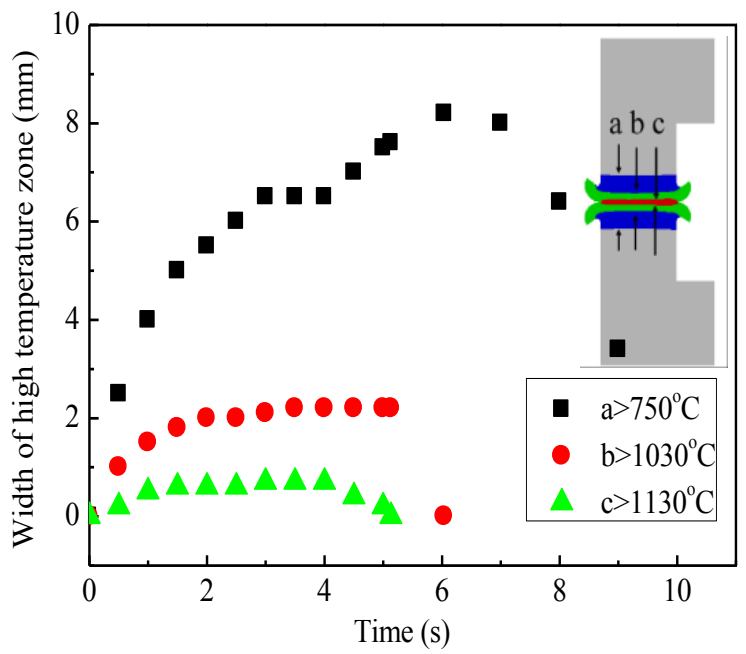

Fig. 10. Variation of the width of high temperature zone with welding time.

Fig. 11 shows the equivalent plastic strain (PEEQ) profiles within the joint at different times during the process. It can be clearly seen that plastic strain is concentrated at the interface and in the flash. It is also shown that the narrowest weldline is present at the centre of the specimen. Through measuring the dimensions of the specimen before and after the experiment, the axial shortening is determined to be about $3.2 \mathrm{~mm}$, which means an error of $3.1 \%$ between experiments and computed axial shortening, indicating a good agreement between experiment and simulation. Fig. 12 shows the final cross-section of the simulated joint at $6 \mathrm{~s}$ and the welded joint. Although the simulated flash is not so similar to the experiment, the simulated PEEQ profile is much similar to the experiment, which provides valuable information about material flow information at the interface.

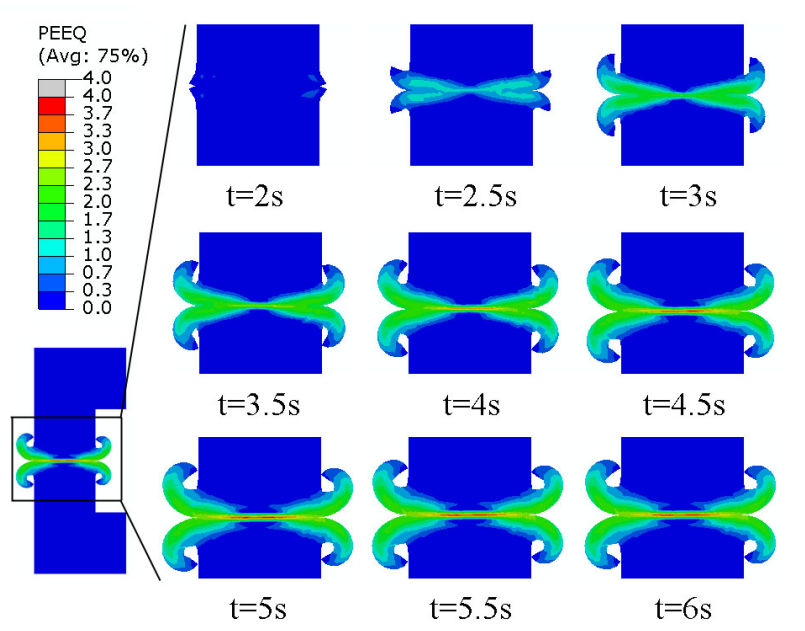

Fig. 11. Equivalent plastic strain profiles at different times.

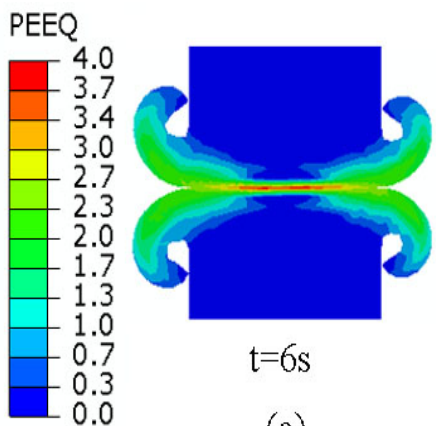

(a)

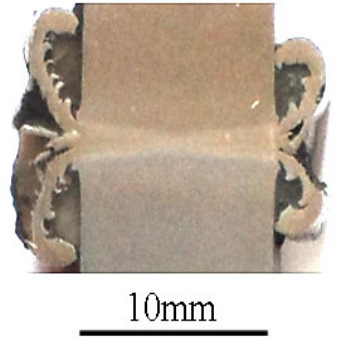

(b)
Fig. 12. Comparison of final cross-sections between simulation (a) and experiment (b)

\subsection{Case 3: LFW}

\section{Case description:}

The 20\# mild steel blocks having dimensions of $45 \mathrm{~mm}$ high, $11 \mathrm{~mm}$ wide and $18 \mathrm{~mm}$ long were LFWed under the welding parameters of oscillation frequency $35 \mathrm{~Hz}$, amplitude $3.25 \mathrm{~mm}$, axial pressure $70 \mathrm{MPa}$ and friction time $3 \mathrm{~s}$.

\section{Geometry and model:}

Based on the previously developed model [20], a 2D model of LFW using ABAQUS was employed as shown in Fig. 13. The specimen has a width of $18 \mathrm{~mm}$ and height of $45 \mathrm{~mm}$, as in the experiments. A rigid surface with the same width as the specimen $(18 \mathrm{~mm})$ was used to model the welding interface, which is a reasonable compromise between computation cost and accuracy. The specimen was partitioned into three areas, where the upper $10 \mathrm{~mm}$ part has a fine mesh size of $0.5 \mathrm{~mm}$, the lower $25 \mathrm{~mm}$ area has a coarser mesh size of $3 \mathrm{~mm}$, and $10 \mathrm{~mm}$ area in the middle has a graduated mesh size. Meshing was performed using 4node quad elements with coupled displacement and temperature, reduced integration and hourglass control. The ABAQUS/Explicit package was used to model the welding phase (extrusion stage) of LFW, while the ABAQUS/Standard package was used for the cooling phase where the specimen does not oscillate any more. 


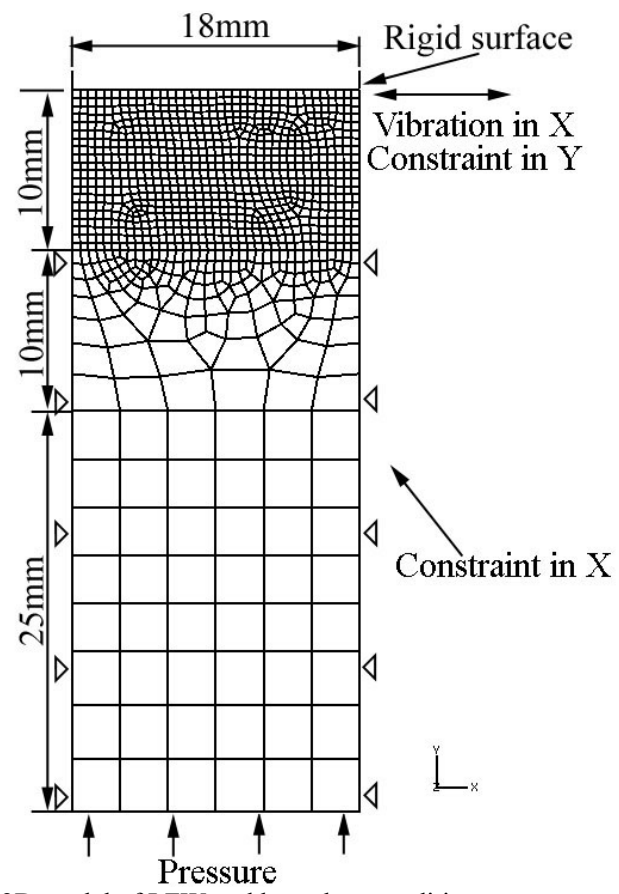

Fig. 13. 2D model of LFW and boundary conditions.

In this model, the external boundary of the lower $35 \mathrm{~mm}$ region was allowed to move only in the $\mathrm{Y}$ direction while constrained in the $\mathrm{X}$-direction. The rigid surface was only allowed to move in the $\mathrm{X}$ direction in a sinusoidal mode with a given amplitude and frequency. To simplify the calculation, a temperature-dependent friction coefficient at the interface was used.

\section{Results and discussion:}

The simulated temperature field at different times during the friction process is shown in Fig. 14. It can be seen that temperature at the interface rises quickly to about $1000^{\circ} \mathrm{C}$ at $1 \mathrm{~s}$, but the high temperature is only limited in the center region. The temperature is about $400^{\circ} \mathrm{C}$ at the corners (at $1 \mathrm{~s}$ ). At this stage no obvious axial shortening is observed as shown in Fig. 15. With further increase of friction time until $2 \mathrm{~s}$, the highest interface temperature increases slowly but the region of high temperature along the interface expands. At the same time, flash is generated and axial shortening becomes apparent as shown in Fig. 15. After about 2s, the interface temperature changes little and axial shortening rate is almost constant, indicating that LFW reaches a quasisteady state where the plasticized metal is continuously extruded. The total unilateral axial shortening is about $2.68 \mathrm{~mm}$ which is in good agreement with the experiment (about $2.72 \mathrm{~mm}$ ).

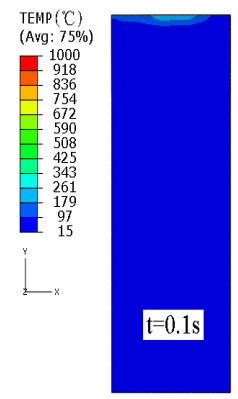

(a)

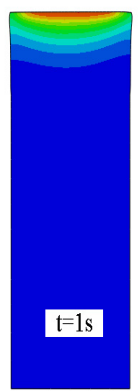

(b)

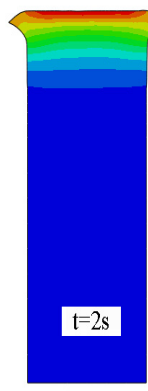

(c)

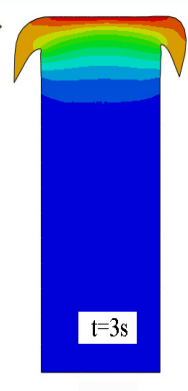

(d)
Fig. 14. Temperature contours of the joint at different welding times of LFW.

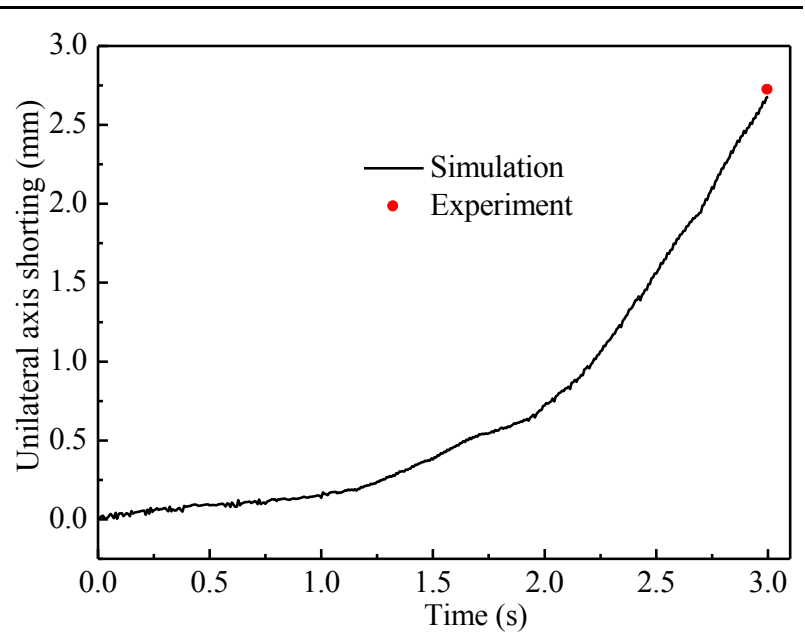

Fig. 15. Comparison between simulated and experimental axial shortening.

Equally good agreement was found for the temperature history of the measured point during welding and cooling phases compared to experiments as shown in Fig. 16. Further to this, the estimated peak temperature is also comparable to the experimental one with an error of $1.6 \%$. In addition, the result also shows the rapid heating and cooling processes during LFW.

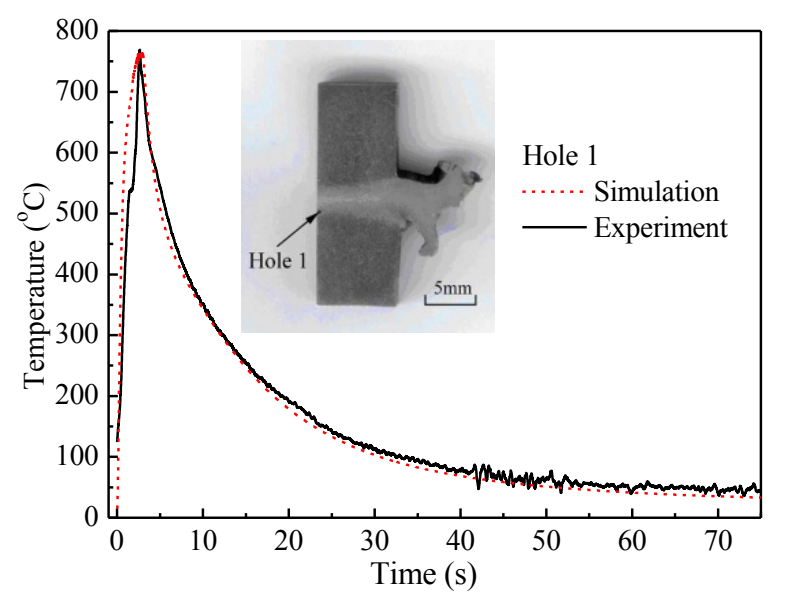

Fig. 16. Comparison between estimated and experimentally recorded temperatures.

\subsection{Case 4: FSW [34]}

\section{Case description:}

FSW experiments were performed on $3.175 \mathrm{~mm}$ thick 2024T3 aluminum alloy sheets at a rotation speed of 300rpm and a welding speed of $60 \mathrm{~mm} / \mathrm{min}$. The tool used was made of $1 \mathrm{Cr} 18 \mathrm{Ni} 9 \mathrm{Ti}$ austenitic steel and had a non-threaded probe with a $5 \mathrm{~mm}$ diameter and $2.5 \mathrm{~mm}$ height as well as nonconcave shoulder with a $15 \mathrm{~mm}$ diameter. The sheets were machined into samples of $200 \mathrm{~mm}$ in length by $95 \mathrm{~mm}$ in width, and butt-welded parallel to the rolling direction. The temperature of certain points at the retreating side of the workpiece were recorded using a K-type thermocouples. The arrangement of the thermocouples at the retreating side is illustrated in Fig. 17. The weld length was $100 \mathrm{~mm}$. The plunge time, dwell time and post-weld air-cooling time were $5.7 \mathrm{~s}, 10 \mathrm{~s}$, and $68 \mathrm{~s}$, respectively. 


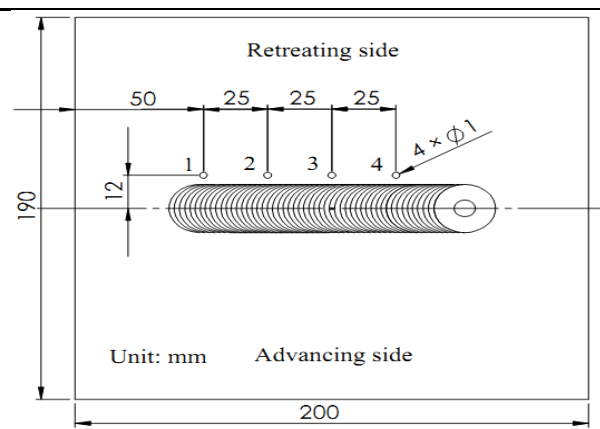

Fig. 17. Arrangement of thermocouples at retreating side of the workpiece.

\section{Geometry and model:}

A three-dimensional numerical model for FSW was developed with ABAQUS/Standard using fully sticking friction. In this model, only one side of the workpiece was considered for modeling as shown in Fig. 18. Heat input was applied as a body heat flux via the subroutine DFLUX. Moreover, contact thermal conductivity between the backplate and workpiece was simplified as convective heat transfer. A convective heat transfer coefficient as a function of temperature and position was applied to the bottom surface of workpiece (contact with the backplate) using the FILM subroutine available in ABAQUS. Furthermore, the fraction of heat transferred into the workpiece was estimated by Equation (7).

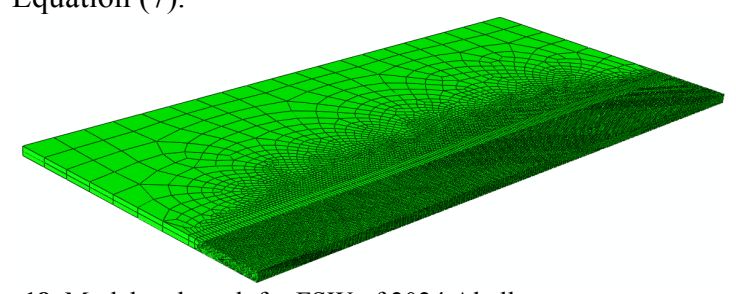

Fig. 18. Model and mesh for FSW of $2024 \mathrm{Al}$ alloy.

\section{Results and discussion:}

The comparison between calculated and experimental temperatures at typical points is shown in Fig. 19. It can be clearly seen that the changing trends of the calculated temperature curves are in agreement with measurements during the entire FSW process, with some difference of the cooling rate being present, implying that the cooling rate has been somewhat underestimated by the model. Further study on the contact thermal conductivity between the backplate and workpiece is essential to obtain the precise temperature field. Moreover, peak temperature is comparable to experiments with a maximum error of about $1.8 \%$.

Fig. 20 shows the transient temperature distribution during the entire FSW process. As the tool probe plunges, the temperature in the material right under the probe rises quickly to about $194^{\circ} \mathrm{C}$ in $2 \mathrm{~s}$ (Fig. 20a). It can be seen from Fig. $20 \mathrm{~b}$ that the temperature under the tool shoulder is about $280^{\circ} \mathrm{C}$ after contacting the workpiece. It is noticed from Fig. 20a-20c that the peak temperature presents a sharp increase from about 194 to $332^{\circ} \mathrm{C}$. Moreover, with increasing welding time, the high temperature region widens from the probe due to heat conduction within the workpiece. From Fig. 20d-20g, it can be shown that the peak temperature in the workpiece is $394^{\circ} \mathrm{C}$ during the entire FSW process and remains constant, which means that there is a thermal balance between heat generation and dissipation within and around the stirred zone at this stage and peak temperature tends to be in a quasi-steady state. At the cooling stage (see Fig. 20g-20j), the peak temperature decreases sharply from 380 to $252^{\circ} \mathrm{C}$ with increasing cooling time from 120.7 to 122.7 s. This means that the fast heat dissipation by heat conduction from the high temperature zone to the cold edges of the workpiece and by the convective heat transfer is relatively large at this stage. However, there is a slight variation in temperature with increasing cooling time after about $140.7 \mathrm{~s}$.

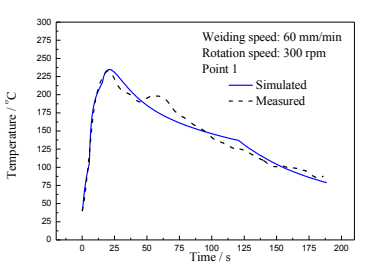

(a)

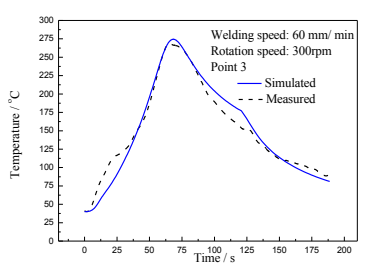

(b)
Fig. 19. Comparison of the simulated and experimental temperature curves at points 1 (a) and 3 (b).

\section{Summary and perspectives}

The simulated temperatures and axial shortening compare well to experiments. However, a lot of simulation work is still necessary for an in-depth investigation of the process. The following open questions will need to be addressed in the future.

\subsection{Excessive element distortion}

FW is a locally-focused large deformation process with extensive plastic deformation occurring in the weld zone. The extruded flash formed in the models is dependent on element deformation in the weld zone, which may cause excessive element distortion. Therefore, this poses a big challenge as just few elements undergo large deformation, especially when the extruded flash is relatively long and thin. Further, element distortion is a more difficult problem to solve in $3 \mathrm{D}$ than in $2 \mathrm{D}$. The commercially available finite element softwares cannot deal to full satisfaction cope with excessive element distortion. Therefore, new algorithms, such as meshless methods, are needed to solve these problems.

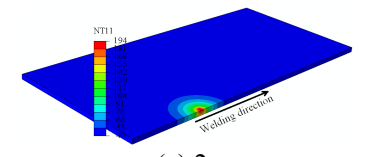

(a) $2 \mathrm{~s}$

(c) $15 \mathrm{~s}$

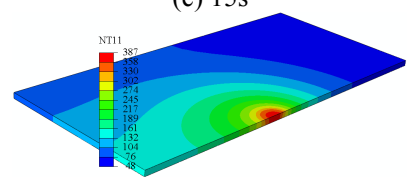

(e) $50 \mathrm{~s}$

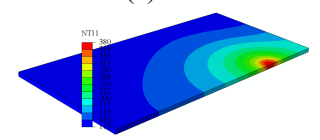

(g) $120.7 \mathrm{~s}$

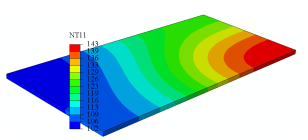

(i) $140.7 \mathrm{~s}$

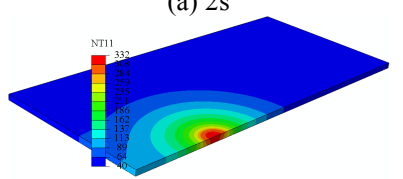

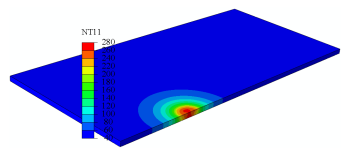

(b) $6 \mathrm{~s}$

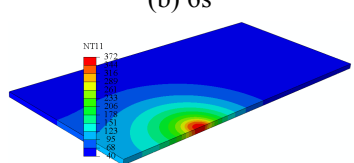

(d) $20 \mathrm{~s}$

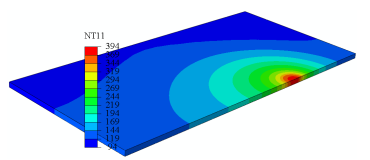

(f) $100 \mathrm{~s}$

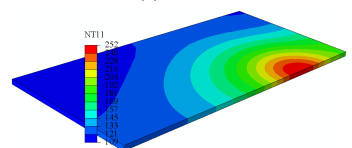

(h) $122.7 \mathrm{~s}$

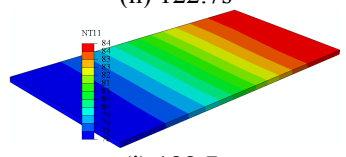

(j) $188.7 \mathrm{~s}$
Fig. 20. Temperature contours at different welding times. 


\subsection{Bonding of the welding interface}

All the simulation work in literature has ignored interface bonding, where the flash is produced by the two components to be welded. If taking the interface bonding into account, more reasonable simulated results may have been reached. Therefore, a more complex contact algorithm is necessary.

\subsection{Flash shapes and ridges}

In the experimental work, the flash with ridges is extruded from the workpieces. Moreover, the frequency of ridges is consistent with movement. Therefore, the shape and ridges of flash depends on the flow behavior of plasticized materials. Up to now, there is no simulation work which has produced the actual shape of flash, let alone the characteristic ridges. Hence, analysis of the flash may be helpful to understand the bonding nature of the FW joint.

\subsection{Dissimilar joints}

The ability to form dissimilar joints is one of the key advantages of FW. However, few related simulation works have been reported. One important reason is that dissimilar materials have different thermal and mechanical properties. Under high friction pressure and interface temperature, the elements in different materials will show different behaviour, and thus dissimilar deformation. This problem will increase the difficulty in simulating contact behavior.

Therefore, a better simulation of FW may rely on, on one hand the solution of the above problems, and on the other hand new algorithms.

\section{Acknowledgements}

The authors would like to thank for financial support the National Natural Science Foundation of China (51005180), the Fok Ying-Tong Education Foundation for Young Teachers in the Higher Education Institutions of China (131052), the Fundamental Research Fund of NPU (JC201233), the Research Fund of the State Key Laboratory of Solidification Processing (Grant No.69-QP-2011) and the 111 Project (B08040).

\section{References}

1. F.D. Duffin and A.S. Bahrani, Wear 26, 53 (1973).

2. A.Vairis and M. Frost, Wear 217, 117 (1998).

3. R.S. Mishra and Z.Y. Ma, Mater. Sci. Eng. R 50, 1 (2005).

4. G. Çam, Int. Mater. Rev. 56, 1 (2011).

5. J.J. Healy, D.J. Mcmullan and S.B. Bahrani, Wear 37, 265 (1976).

6. G.J. Bendzsak, T.H. North and Z. Li, Acta Mater. 45, 1735 (1997)

7. O.T. Midling and Ø.Grong, Acta Metall. Mater. 42, 1595 (1994).

8. A. Sluzalec, Int. J. Mech. Sci. 32, 467 (1990).

9. V.R. Davé, M.J. Cola and G.N.A. Hussen, Weld. J. 80, 246 (2001).

10. M. Soucail and Y. Bienvenu, Mater. Sci. Eng. A 220, 215 (1996).

11. L. D'Alvise, E. Massoni and S. Walle, J. Mater. Process. Tech. 125-126, 387 (2002).

12. L.W. Zhang, C.D. Liu, S.A. Qi, Y.S. Yu, W.H. Zhu, S. Qu and J.H. Wang, J. Phys. IV 120, 681 (2004).

13. C.J. Bennett, T.H. Hyde and E.J. Williams, P. I. Mech. Eng. L. J. Mat. 221, 275 (2007).

14. L. Wang, M. Preuss, P.J. Withers, G. Baxter and P. Wilson, Metall. Mater. Trans. B 36, 513 (2005).

15. B. Granta, M. Preuss, P.J. Withers, G. Baxter and M. Rowlson, Mater. Sci. Eng. A 513-514, 366 (2009).

16. A. Vairis and M. Frost, Mater. Sci. Eng. A 292, 8 (2000).

17. J. Sorina-Müller, M. Rettenmayr, D. Schneefeld, O. Roder and W. Fried, Comp. Mater. Sci. 48, 749 (2010).

18. R. Turner, J.C. Gebelin, R.M. Ward and R.C. Reed, Acta Mater. 59, 3792 (2011).

19. J. Tao, T.C. Zhang, P.T. Liu and J. Li, Mater. Sci. Forum 575-578, 811 (2008).
20. W.Y. Li, T.J. Ma and J.L. Li, Mater. Design 31, 1497 (2010).

21. J. E. Gould and Z. Feng, J. Mater. Process. Manuf. Sci. 7, 185 (1999).

22. H. Schmidt, J. Hattel and J. Wert, Modelling Simul. Mater. Sci. Eng. 12, 143 (2004).

23. M.Z.H. Khandkar, J.A. Khan and A.P. Reynolds, Sci. Technol. Weld. Joi. 8, 165 (2003).

24. P. Ulysse, Int. J. Mach. Tool. Manuf. 42, 1549 (2002).

25. T.U. Seidel and A.P. Reynolds, Sci. Technol. Weld. Joi. 8,175 (2003).

26. R. Nandan, G.G. Roy, T.J. Lienert and T. Debroy, Acta Mater. 55, 883 (2007)

27. P.A. Colegrove and H.R. Shercliff, Sci. Technol. Weld. Join. 8, 360 (2003).

28. J.H. Cho, D. Boyce and P. Dawson, Modelling Simul. Mater. Sci. Eng. 15, 469 (2007).

29. S. Guerdoux and L. Fourment, Modelling Simul. Mater. Sci. Eng. 17,1 (2009).

30. ABAQUS 6.11 Documentation, Dassault Systèmes Simulia Corp., Providence, RI, USA, 2011.

31. Z. Zhang, J. Mater. Sci. 43,5867 (2008).

32. W.Y. Li, F.F. Wang, Mater. Sci. Eng. A 528, 5921 (2011).

33. F.F. Wang, W.Y. Li, J.L. Li and D.L. Gao, 2nd International Symposium on computer-Aided Welding Engineering, JiNan, China, pp. 426-439 (2012).

34. Z.H. Zhang, W.Y. Li, J. Han and J.L. Li, China Welding 20, 76 (2011) 